\title{
Caracterización de los canales de comercialización de limón ecológico malagueño*
}

Cada vez en mayor medida al productor hortofrutícola no le es suficiente producir con calidad y en cantidad, sino que debe adaptarse a un contexto en el que el consumidor demanda información acerca no solo del producto, sino también sobre la forma en la que este ha sido obtenido, manipulado y comercializado. Además, en un contexto de creciente competencia, el productor se está viendo obligado a tratar de diferenciar su producción a través de lo que podrían denominarse «ventajas en diferenciación» basadas en atributos de confianza que vienen avalados por el cumplimiento de toda una serie de normativas y/o protocolos de producción y comercialización, entre las que se encuentra la Certificación de Agricultura Ecológica.

En la actual crisis de la citricultura malagueña en general, del limón en particular, esta posibilidad de diferenciación de la producción pareciera que es una de las escasas opciones que los productores tienen a la hora de lograr un incremento de los precios obtenidos por sus producciones, mejorando las cuentas de la explotación. De las distintas opciones existentes (cumplimiento de protocolos de buenas prácticas agrícolas - GAP-, Producción Integrada, Denominación de Origen, entre otras), es la Agricultura Ecológica

\footnotetext{
* Este estudio se enmarca en los resultados del Proyecto de Excelencia «Caracterización y diagnóstico del complejo agrocomercial limonero malagueño en un contexto de crisis» (P07HUM-02727), financiado por la Consejería de Innovación, Ciencia y Empresa de la Junta de Andalucía.
} 
la que, hasta el momento, está más extendida entre los citricultores malagueños dedicados al limón.

No obstante, el consumo de productos ecológicos en España en general, y en Andalucía en particular, está aún muy poco desarrollado, y menos aún el consumo de limón ecológico, pese a los intentos por parte de las diferentes Administraciones de fomentar el consumo interno de productos con esta certificación. Es así que frente a unas cuotas de mercado de productos ecológicos que oscilan entre el 4,5\% de Suiza y el 2,5\% de Gran Bretaña, en España la cifra fue de tan solo el 0,1\% en 2006 (Padel et al., 2008). Otro dato que apunta en esta misma dirección es el correspondiente al gasto medio por persona, que en 2007 fue de tan solo $12 €$ al año en nuestro país, una cifra que contrasta con los $102 €$ de los suizos (los que más gastan anualmente en este tipo de producto), o con la media de la Unión Europea, 27€. Es Andalucía una de las principales productoras y consumidoras de productos ecológicos, pero incluso en ella, el limón no es uno los productos ecológicos comprados más frecuentemente, al tiempo que sus perspectivas de aumento de consumo no son demasiado elevadas.

\section{FACTORES QUE DIFICULTAN LA ADOPCIÓN DE LA OPCIÓN ECOLÓGICA}

Como hemos indicado, la obtención de la Certificación de Agricultura Ecológica es una de las escasas alternativas que tienen los productores malagueños a la hora de diferenciar su producción, pero su adopción se está viendo dificultada por toda una serie de problemas, que podrían agruparse en dos categorías: de actitud por parte de los productores, y económicos.

El primer grupo se deriva en gran medida de dos características definitorias de los citricultores malagueños: una estructura por edad muy envejecida, y una dedicación a tiempo parcial a la agricultura, que determinan una escasa motivación para realizar la transición a ecológico. En este sentido, la falta de una renovación generacional entre los agricultores, la escasa información que poseen sobre la agricultura ecológica y sobre el proceso de certificación, y la falta de liderazgo y de carácter emprendedor son elementos que han sido identificados como debilidades a la hora de afrontar este proceso de cambio (di Paula, 2009). Debe tenerse en cuenta que la agricultura ecológica ha sido considerada como un medio de innovación en el medio rural, indicándose que «la $\mathrm{AE}$ es una forma innovadora de visualizar y practicar la agricultura. Esta fuerza innovadora se manifiesta a sí misma en varios aspectos. La AE es una innovación compleja, que requiere un alto nivel de formación y unos requerimientos técnicos bajos» (Pugliese, 2001). 
La importancia de la capacitación adquiere su verdadera importancia al considerar que el productor ya no solo debe saber producir, sino que si desea realizar la conversión a ecológico además debe conocer los requisitos legales de gestión obligatorios, y las condiciones agrarias y medioambientales satisfactorias que estipulan los artículos 4 y 5 de los anexos III y IV del Reglamento (CE) 1782/2003, así como las normas relativas a la seguridad laboral basadas en la legislación comunitaria. Dada la estructura por edad de los citricultores malagueños dedicados al limón (en 1999, solo el 17\% tenía menos de 40 años, y el 38\% más de 60), y la influencia negativa que en el Valle del Guadalhorce las edades avanzadas tienen para la adopción de innovaciones (Ocaña Ocaña y Larrubia Vargas, 1993), las posibilidades de que las cohortes más envejecidas - las más numerosas- se decidan por la conversión no son demasiado elevadas.

Otro elemento que creemos que dificultará el acceso a la agricultura ecológica es la obligación de formarse específicamente que se impondrá, según el II Plan de AE de la Junta de Andalucía, a los jóvenes agricultores que comiencen a producir bajo métodos ecológicos, y a los agricultores ya instalados que pretendan reconvertirse a producción ecológica; es más, esta capacitación será condición necesaria para la concesión de nuevas solicitudes de Ayudas Agroambientales (que están empleándose en gran medida para pagar los costes de la certificación). En un contexto de virtualmente nula sustitución generacional y de envejecimiento progresivo del plantel de productores, esta exigencia puede convertirse en un freno para la reconversión.

El otro conjunto de factores que frenan la adopción de la certificación ecológica como vía de diferenciación de la producción es el relacionado con el coste, desde el momento en que el tamaño tan reducido de la mayor parte de las explotaciones limoneras malagueñas dificulta asumir el coste de la certificación (alrededor de $130 €$ por hectárea, más el coste de las tomas de muestras y visitas de los técnicos de la Certificadora). A ello hay que añadir los mayores precios de los insumos permitidos en la opción ecológica (alrededor de un $10 \%$ más que en la opción de convencional [Navarro Rodríguez y Larrubia Vargas, 2002]), máxime en el contexto actual de bajos precios de la fruta que, para producciones pequeñas, no asegura la rentabilidad. Además, se debe tener en cuenta que el agricultor debe hacer frente a estos gastos desde el mismo momento en que realiza la inscripción, pero que no podrá vender su limón como ecológico hasta pasado un periodo de tiempo, un periodo de reconversión, de duración variable (entre 6 meses y dos años).

De cualquier forma, se ha indicado (Domínguez Gento, 2008) que el sobreprecio de los cítricos ecológicos con respecto a los convencionales en la 
Unión Europea (como veremos, principal destino de la producción malagueña) es de un $65 \%$ de media, alcanzando el $125 \%$ en Alemania y el $78 \%$ en Francia. Por tanto, es necesario prestar atención a los mercados a los que se dirige el limón ecológico malagueño.

\section{LA COMERCIALIZACIÓN DEL LIMÓN ECOLÓGICO MALAGUEÑO}

Históricamente la mayor parte de la producción andaluza de productos ecológicos se ha dirigido al exterior, por lo que sus estructuras productivas y de distribución están en gran medida adecuadas a este mercado, en detrimento del interno. En el caso concreto de los cítricos, en 2008 se exportó —básicamente a Alemania, Francia, Reino Unido y Holanda- el 76,6\% de la producción de los operadores certificados por la Asociación CAAE —la certificadora de mayor implantación en Andalucía-, vendiéndose en el mercado nacional el 23,4\% restante. En esta desproporción entre los envíos realizados al exterior y las ventas en el mercado interno subyace el hecho de que el mercado español de productos ecológicos ha estado (y aún está) escasamente desarrollado, recordemos las cifras de consumo a las que hicimos referencia en párrafos anteriores; en este sentido, el hecho de que el consumo es el «cuello de botella» de la producción ecológica andaluza es reconocido por la propia Dirección General de Agricultura Ecológica de la Junta de Andalucía, de ahí que desde ella se estén promoviendo de manera activa el consumo en general, y los canales directos - canales cortos- de distribución en particular.

Estos canales cortos se caracterizan por que las funciones realizadas por los comercializadores son asumidas por los propios productores, o por cooperativas de consumidores; se ofrece así información clara sobre el origen de los productos, y se fomentan las relaciones personales e inmediatas entre productor y consumidor. Para el productor, el empleo de estos canales de comercialización supone la obtención de un mayor valor añadido, reduciendo su dependencia de los mercados externos y de los intermediarios. Este tipo de canal, además, tiene más variedad en lo relativo a la tipología de puntos de venta y formas de comercialización, y, desde el momento en que la necesidad de intermediarios entre productor y consumidor es menor -inexistente, en los casos extremos, presenta algunas posibilidades potenciales de venta para los pequeños productores que, habiéndose decidido por la conversión a ecológico, no cuentan con acceso a otros canales de comercialización.

En 2008 la Junta de Andalucía preparó una relación de puntos de venta de productos ecológicos, discriminados por provincia, cuyo objetivo era recoger 
información de las empresas andaluzas que venden productos ecológicos directamente al consumidor, para así fortalecer el consumo y el conocimiento del sector, un listado que se resuelve como el registro más sistemático de canales cortos consolidados de los que disponen los productores malagueños de limón ecológico. Dada la importancia potencial que se le asignan a los canales cortos de distribución parece conveniente estimar la capacidad que tienen a la hora de dar salida a la producción malagueña de limón ecológico; para ello, hemos realizado una serie de entrevistas sobre la base del listado anterior, y que apuntan a que los canales cortos de comercialización no son sino una opción muy secundaria de comercialización.

Así, el volumen que pueden mover las tiendas especializadas es poco menos que testimonial, menos de una tonelada al año; también extremadamente escaso es el volumen de limón ecológico que se comercializa a través de la Asociación de Consumidores «La Breva», situada en la capital provincial y que trabaja con el sistema de cestas fijas, que ha sido cifrado por los entrevistados en alrededor de los 600 kilos anuales. Tampoco los cinco mercados ecológicos que se celebran en la provincia son capaces de dar cuenta de la comercialización de una porción significativa del limón ecológico producido en Málaga. Tal y como las entrevistas han indicado, esta iniciativa está más orientada hacia las producciones hortofrutícolas, por su mayor diversidad de producción y demanda por parte del consumidor, pero se resuelve como incapaz de absorber una porción significativa de la producción de limón.

Por último, y asociado a las nuevas tecnologías, progresivamente se está abriendo un nuevo canal de comercialización directa, centrado en la Web, que tiene como protagonista a empresas de venta directa por Internet. No contamos con una cifra exacta del volumen de limón vendido, pero de las entrevistas mantenidas se deduce que este no es demasiado elevado, en cualquier caso insuficiente como para considerar este canal como con capacidad de dar salida a una porción significativa de la producción ecológica de la provincia.

Además de los que acabamos de reseñar, en Andalucía se está registrando la aparición de otro canal que está aumentando el consumo de productos ecológicos, en el marco del denominado Programa de Consumo Social de alimentos ecológicos, impulsado desde 2005 por la Dirección General de Agricultura Ecológica. Pero si bien es cierto que es, tal y como se reconoce desde el sector, una iniciativa de gran interés para el conjunto de la agricultura ecológica andaluza, paralelamente se intuye que su impacto en el limón probablemente será menor — residual — en comparación con el que se obtendrá en otros productos ecológicos, puesto que su demanda se restringe a su empleo como aliño o aditamento opcional. 
A la vista de todo lo anterior, resultaría evidente que, debido al escaso volumen de demanda - y por tanto de ventas- que suponen, los canales cortos de comercialización no pueden sino considerarse como alternativas a las ventas realizadas por otras vías, ya sean dirigidas al mercado interno o, preferiblemente, a la exportación.

En este sentido, la existencia de canales de comercialización nacionales consolidados para el limón convencional decididamente podría ser aprovechada para la comercialización del ecológico. Sin embargo, la situación existente a finales de 2009/comienzos de 2010 en lo relativo a las ventas de limón ecológico malagueño en grandes superficies no es muy diferente de la que hasta aquí hemos reseñado. Así, en una conocida cadena de hipermercados de capital francés este tipo de producto estaba disponible en un único centro de la provincia, pero se retiró por falta de demanda, mientras que en otra conocida cadena de centros comerciales de origen español sí es posible encontrar limón ecológico, pero no de procedencia malagueña; además el volumen comercializado es poco menos que testimonial, alrededor de 4 kilos mensuales, en presentaciones de bandejas de 500 gramos. Con estas cifras, resulta patente que la demanda articulada a través de estos tipos de establecimientos es incapaz también de absorber la producción de limón ecológico malagueño.

Visto el volumen tan reducido de limón ecológico que, hasta el momento, se mueve en el mercado interno, no debe extrañar el hecho de que sea la exportación la principal salida de la producción de la provincia, una exportación que se articula a través de tres canales fundamentales: la venta a través de cooperativa, a través de comercializadoras, y a través de procesos de integración vertical.

La primera de las posibilidades está representada por Agrolimón S.C.A., localizada en la zona productora de la Axarquía. La labor de la cooperativa en relación con la venta del producto ecológico no hace sino reproducir las pautas correspondientes al convencional, esto es, concentra la oferta de un número elevado de muy pequeños productores, y diversifica los destinos, añadiendo valor añadido a la producción a través del calibrado, envasado y etiquetado con trazabilidad de la fruta.

El destino del limón ecológico producido por sus socios es variable, aunque la mayor parte de la producción se destina al mercado externo; básicamente Francia, en menor medida a Alemania y Gran Bretaña. La venta en estos mercados se realiza a través del gerente de la cooperativa (que hace las veces de comercial), enviándose por carretera el producto al destino. Y, al contrario de lo que ocurre con la fruta convencional, que se comercializa parcial- 
mente con marca propia (Limonsur), el limón ecológico es envasado en cajas de 5 kilogramos, sin marca, tan solo con el etiquetado «a la caja» exigido para este tipo de producto.

El otro canal de comercialización que emplea la Cooperativa para dar salida a su producción ecológica es la venta a empresas comercializadoras, tanto de la provincia de Málaga — como Green-Food, Eurofresh—, como de fuera -Campiña Verde, Ecolevante, o Solfruit o Hyperion, comercializadores europeos- Alguna de estas comercializadoras exigen que el limón que adquieren les sea enviado envasado en las instalaciones de la cooperativa en sus propias cajas, mientras que a otras se les sirve el limón en cajas sin marca, tan solo etiquetadas de la manera anteriormente reseñada.

El segundo canal de exportación son las diferentes comercializadoras que, como acabamos de apuntar, trabajan con limón ecológico de la provincia. Ejemplos de este tipo de empresas surgidas en la propia zona productora, y centradas en la comercialización de cítricos (no solo limón), son Bio Costa del Sol, o Pizarra Export, localizadas en el municipio de Pizarra. Estas empresas realizan compras a agricultores ecológicos de la zona -ellos mismos no son productores-, procediendo a la venta, en mercados europeos, de la fruta, ya sea con marca propia, ya sea con la marca del comprador, disponiendo para el acondicionamiento de la fruta de instalaciones propias en el municipio.

Junto a ellas hay otro conjunto de comercializadoras, también malagueñas, pero localizadas fuera del Valle del Guadalhorce, y más centradas en la comercialización de otros productos ecológicos. Una de las más representativas es Eco Green-Food Import-Export. Esta empresa, establecida en 2005 (aunque sus integrantes tienen experiencia en ecológico - aguacates- desde comienzos de los noventa), tiene el grueso de su actividad orientado a subtropicales y hortalizas, envasando el producto acorde a los requerimientos exigidos por el cliente en cuanto a formato y etiquetado. Esta empresa comercializó en 2008 aproximadamente 150.000 kilos de limón ecológico, fruta producida por 28 productores de la provincia de Málaga (y también, como hemos indicado, por una parte de los asociados a Agrolimón). El destino final es la exportación, siendo los principales países de destino Francia, Alemania, Suiza y Holanda.

Por último, la tercera vía de exportación está compuesta por un reducido conjunto de productores que han logrado vender su limón ecológico directamente al exterior, a través de un proceso de integración vertical; entre ellos podemos destacar el caso de una familia de productores de Alhaurín de la Torre, con varias décadas de experiencia en el cultivo de cítricos, que durante la 
década de los noventa deciden dar el paso de la conversión a ecológico. En la actualidad cuenta con aproximadamente 18 hectáreas de limón ecológico, en las que producen de media 600/700 toneladas anuales de limón; de ellas, un porcentaje que varía, según el año, entre el $85 \%$ y el $75 \%$ se vende en fresco, en su mayor parte al mercado externo. En relación con ello, es interesante indicar que los contactos con los compradores son nuevos, en el sentido de que no se han empleado los correspondientes a limón convencional, puesto que no exportaban con anterioridad. La asistencia a ferias, convenciones, etc., se ha resuelto en este caso como la vía principal para la apertura de contactos. Los destinos son Alemania y Francia, principalmente, mercados donde la venta se hace sin intermediarios a las grandes cadenas de distribución. Recientemente han dado un paso más en la integración vertical de la actividad con la puesta en marcha de instalaciones para el tratamiento de la fruta, de forma que tienen capacidad para vender el limón en el formato que el cliente requiera — granel, enmallado...-, con la marca del comprador, si se exige, o incluso con marca propia. La manera de venta es a demanda, y el precio puede ser puesto en destino, o a pie de finca.

A la vista de todo ello, la venta del grueso de la producción ecológica pasa por el mercado internacional, a través de diferentes canales de comercialización. Este es un destino al que tan solo un número reducido de productores están siendo capaces de llegar a través de modestos procesos de integración vertical, merced a los cuales logran colocar su producción y, progresivamente, la de otros operadores certificados. Junto a la opción cooperativa - de radio comarcal, y localizada fuera de la principal zona de producción de limón ecológico-, el otro canal de acceso al mercado internacional son las comercializadoras «tradicionales». Una opción que, finalmente, coloca a los pequeños productores en una situación de dependencia parecida a la correspondiente al limón convencional, si no por los precios obtenidos —más elevados-, sí por su escasa capacidad de negociación de estos, puesto que, dada la fragmentación de la oferta, y la escasa capacidad de absorción que suponen los canales cortos, la única opción de venta que queda es el mercado exterior, al que no todos tienen acceso directo. Por tanto, el asociacionismo agrario, o, al menos, los acuerdos entre productores a la hora de concentrar la oferta, se resuelven como necesarios también en la opción ecológica a la hora de fortalecer las opciones de los productores para obtener precios razonables por la única oferta diferenciada de limón de la tercera zona productora española. 


\section{BIBLIOGRAFÍA CITADA}

Armesto López, X. A. (2007): "El concepto de agricultura ecológica y su idoneidad para fomentar el desarrollo rural sostenible”. Boletín de la AGE, 43, pp. 155-172.

Domínguez Gento, A. (2008): La citricultura ecológica. Sevilla, Consejería de Agricultura y Pesca, Junta de Andalucía.

Navarro Rodríguez, S., y Larrubia Vargas, R (2002): "La agricultura ecológica: producción y comercialización en la provincia de Málaga". Baetica, 24, pp. 113-148.

Ocaña Ocaña, C. y Larrubia Vargas, R. (1993): Agricultura y espacio metropolitano: Málaga y el Bajo Guadalhorce. Málaga, Servicio de Publicaciones de la Universidad de Málaga. 169 pp.

Padel, S. et al. (2008): "The european market of organic food in 2006", en H. Willer, M. Yussefi-Menzler y N. Sorensen, N. (eds.) (2008): The world of organic agriculture. Statistics and emerging trends 2008. IFOAM-FiBL pp. 1 31-139. Disponible en http://orgprints.org/13123/ (consultado 06/09/2011).

Paula, V. di (2009): La producción ecológica en la provincia de Málaga: tendencias y perspectivas. Málaga, Diputación Provincial de Málaga.

Pugliese, P (2001): "Organic farming and sustainable rural development: a multifaceted and promising convergence". Sociologia Ruralis, 41/1. pp. 112-130.

Juan José Natera Rivas

Facultad de Filosofía y Letras. Universidad de Málaga 